\title{
Application of Polynomial Regression Analysis in Evaluating the Techno-Economic Performance of DSPV Transformers
}

Bonginkosi Allen Thango*, Jacobus Andries Jordaan, Agha Francis Nnachi

Department of Electrical Engineering, Tshwane University of Technology, Emalahleni, 1034, South Africa

\begin{tabular}{l} 
A R T I C L E I N F O \\
\hline Article history: \\
Received: 22 December, 2020 \\
Accepted: 08 February, 2021 \\
Online: 17 March, 2021
\end{tabular}

Keywords:

Transformers

Distributed Solar Photovoltaic

Total Ownership Cost

\begin{abstract}
A B S T R A C T
To this extent, the delineation of techno-economic evaluations for transformers becomes more intricate through a lens of Distributed Solar Photovoltaic (DSPV) market in South Africa. Essentially, the transformer price and loss evaluation techniques should be tailored for calculating the Total Ownership Cost (TOC) of transformers facilitating decentralized energy systems. In South Africa, the traditional coal power generation and renewables operate concurrently under liberalized energy markets but have distinct operational requirements and therefore have distinct methods for evaluating their generating states, service loss costs and TOC. As a result, their techno-economic evaluations should be different. In this work, new formulae have been developed to contemplate on a comprehensive technique for calculating the transformer prices and losses necessitated to estimate the cost of service losses and TOC for DSPV transformers. These formulae are based on experimental studies undertaken on a fleet of DSPV transformers ranging from 1.25 to 250MVA. In order to substantiate these new formulae, 4 case studies have been presented. The calculated losses and associated cost results against the pragmatic values from the case studies yield an error of estimation of less than $1 \%$ and $2 \%$ respectively in all cases. Further, these results are used to calculate the cost of losses and TOC using a methodology that has been proposed in previous work exclusively for power producers who are proprietors of DSPV generation systems.
\end{abstract}

\section{Introduction}

Transformers make up some of the fundamental powerconsuming equipment in the Distributed Solar Photovoltaic (DSPV) systems. The use of transformers has enabled the renewable energy market to grow steadily, with the transformer market expected to increase annually by approximately $16 \%$ between 2019 and 2020 [1]. The increased attention by the government to broaden investments in renewable energy resources to satisfy environmental and sustainable objectives is the main driver of this market growth [2]-[5].

The cost-effective measures derived from the choice of enhanced energy efficiency criterion at the time of installation and commissioning of new transformers or replacement of existing units can culminate in a considerable amount of losses decreasing and some benefits for DSPV systems. Contemporary energyefficient transformers are designed to operate at minimum total losses viz. no-load loss, load loss and auxiliary loss. Transformers "Corresponding Author: Bonginkosi Allen Thango, thangotech@gmail.com manufactures have modernised the manufacturing procedures and new type of optimised core material with thinner laminations [6], [7] to bestow cost-productive and energy-efficient transformers to utility owners such as the Independent Power Producers (IPP's). Substantially, they reduce power consumption and therefore reduce energy generation and the resultant greenhouse gas (GHG) emissions. Therefore, as renewable energy power systems investment keeps on growing, IPP's are to a larger and larger degree fascinated in installing energy-efficient transformers in their power networks.

The initial purchase price of energy-efficient transformers is relatively high; however, they consume less power during their intended service life in comparison to low-efficiency transformers. The choice regarding buying cheap but high loss transformer or a more expensive, with low loss transformer is mainly a lucrative one. The clarification for picking out one transformer over another should be founded on the initial purchase price plus the operational cost experienced over its intended service lifetime [8], [9], and [10]. A habitual procedure for evaluating the service lifetime cost 
of operation, and consequently the best purchase decision for transformers, is established on a service lifetime loss evaluation procedure that gives the ensuing Total Ownership (TOC $-Z A R)$ of Transformers. It is pivotal to appreciate that each power utility's operation conditions, to the extent that the transformers service lifetime loss evaluation methods are concerned, could be not similar. Even within one country, different power utilities may have unique operation objectives and financial goals. For instance, service lifetime loss evaluation procedure for DSPV systems engrosses a thorough knowledge of the generation profile based on the availability of solar irradiation and estimation of the incurred losses as a result of this effect and other factors including possible additional losses due to harmonic currents.

On the other hand, for wind plants, a comprehensive understanding of the factors like wind output speed and generation hours per annum are essential [11] - [12]. In the mainstream, the transformers service lifetime loss evaluation method regarded scheming device where its execution predominantly depended on every renewable energy power system's circumspection. Therefore, there might be significant inconsistencies when describing electrical network cost and load factors employed loss evaluation procedures. These inconsistencies are based on the unique operation objectives and financial goals allotted by each renewable energy power system and the extent of analysis involved. The constituents undertaken in the procedures include the network's load profile, rate of interest and energy tariffs, and other operational expenditures.

This work is an extension of previous work presented in [13]. The aim of the current work is to develop a new formulae for establishing the cost of various transformers and their associated losses to advance the adjudication of competing transformer bid offers, evaluation of techno-economic parameters of transformers for new DSPV projects, defer replacement and strategic planning to supersede existing units in service. The advancements include: a) a new formula for calculating the transformer price per $M V A$ and $Z A R / M V A$ of a wide range of transformers from $1.25-$ 250MVA, (b) a new formula for calculating the associated no-load and load losses per MVA and Watts/MVA. The proposed loss evaluation procedure by the authors and associated annualised cost of energy, interest rate, service lifetime and the generating and non-generating state factors have been adopted from previous work presented in [13] in order to evaluate the cost of service losses and TOC of the studied transformers. The results yield an error of estimation of less than $1 \%$ and $2 \%$ for the losses and associated cost; and the TOC respectively.

\section{Economic Evaluation of Transformer Service Lifetime}

The service lifetime loss evaluation of transformers is a procedure employed by transformer manufactures and IPP's to account for the summation of the present cost of unit of electrical energy in kilowatt $(k W)$ of the transformer over its intended service lifetime. The loss of electrical power in transformers are defined as the no-load loss $\left(P_{N L}-k W\right)$, the load loss $\left(P_{L L}-k W\right)$, and the auxiliary loss $\left(P_{A u x}-k W\right)$. Therefore, when determining these transformer losses, their assessment is established on the basis of the plant's energy cost $\left(E_{C}-Z A / k W\right)$ and energy demand $\left(E_{D}-Z A R / k W\right)$ constituents. The energy cost $\left(E_{C}-Z A / k W\right)$ is the present cost of energy unit of electrical energy in kilowatt $(k W)$ that will be consumed by the transformer over its service lifetime within the renewable power plant [14]. Moreover, the energy demand $\left(E_{D}-Z A R / k W\right)$ is the present value of electrical energy in kilowatt $(k W)$ of installing an electrical load to service the energy used by the losses [14]. Insofar, the energy and demand factors of the losses are the predominant factors in the procedure of determining the cost of the renewable power plant and energy necessary to supply the service lifetime losses of transformers.

The energy and demand constituents are properly annualised to give the total loss value $(Z A R / k W)$ which include the sum of equivalent discounted value of unit of electrical energy in kilowatt $(\mathrm{kW})$ of the transformer over its intended service lifetime. The loss evaluation procedure ensue the discounted Total Cost of Losses $(T C L-Z A R / k W)$ of the transformer over its intended service lifetime. The TCL of transformers can be expressed as follows in Eq. (1) as an arithmetic sum of the cost of, the cost of no-load loss $\left(C_{N L}-Z A R\right)$, the cost of load loss $\left(C_{L L}-Z A R\right)$, and the cost of auxiliary loss $\left(C_{A u x}-Z A R\right)$.

$$
T C L=C_{N L}+C_{L L}+C_{A u x}
$$

The rate $\left(F_{N L}\left(E_{D}, E_{C}\right)-Z A R / k W\right)$ that represents the cost of unit of electrical energy in kilowatt $(k W)$ of the transformer noload loss over its intended service lifetime is employed as follows in Eq. (2).

$$
C_{N L}=F_{N L}\left(E_{D}, E_{C}\right) \times P_{N L}
$$

The rate $\left(F_{L L}\left(E_{D}, E_{C}\right)-Z A R / k W\right)$ the represents the cost of nit of electrical energy in kilowatt $(k W)$ of the transformer load loss over its intended service lifetime expressed is employed as follows in Eq. (3).

$$
C_{N L}=F_{L L}\left(E_{D}, E_{C}\right) \times P_{L L}
$$

The rate $\left(F_{A u x}\left(E_{D}, E_{C}\right)-Z A R / k W\right)$ that represents the cost of unit of electrical energy in kilowatt $(k W)$ of the transformer auxiliary loss over its intended service lifetime is employed as follows in Eq. (4).

$$
C_{\text {Aux }}=F_{\text {Aux }}\left(E_{D}, E_{C}\right) \times P_{\text {Aux }}
$$

\subsection{Transformer TOC Method}

The total ownership Cost $(Z A R)$ of transformers in therefore attained using the initial buying price $(B P-Z A R)$ of the transformer and its TCL as expressed in Eq. (5) [15] - [16].

$$
T O C=B P+T C L
$$

The TOC's value is an economic estimation employed to give the IPP's and investors with the overall operational and maintenance costs of their transformer expenditure. Additionally, the TOC is a planning device that can be useable according to the following conditions.

\section{a) Optimization of the transformer design philosophy}

It is widely accepted that the transformer losses in the renewable energy power systems is relatively high [17]-[18], and 
the manufactures design philosophies must be imbedded with data such as a complete harmonic spectrum to design the most optimised offers. The effect of such implementation will inescapably increase the transformer price. In any event, the TOC model fortifies the reality that reducing transformer losses with expensive and lower losses transformer would comprehensively indicate the reduction of the transformer operational, maintenance and consequently the ownership cost. The consequence of this approach will have significant energy preservation for the IPP's and put off the necessity for renewable power utility energy tariffs increase.

\section{b) Competing transformers during a bid window}

The service lifetime loss evaluation procedure and the resulting TOC allows the IPP's to appraise competing transformer manufacturer's offerings in preparing to the highest degree the excellent choice of buying transformers between competing transformers and so affirm buying of the most economical units. Employing the rates $F_{N L}\left(E_{D}, E_{C}\right), F_{L L}\left(E_{D}, E_{C}\right)$ and $F_{A u x}$ that represents the cost of a unit of electrical energy in kilowatt $(k W)$ of the DSPV transformer losses over their intended service lifetime, the financial reward of high initial buying price but lowloss units can be easily compared with lower initial buying price and high-loss units.

\section{c) Estimation of the transformer service lifetime}

The service lifetime loss evaluation procedure give knowledge to the IPP's of the estimated and most suitable time to replace an existing transformer with more economical and lower losses. This knowledge takes into consideration the financial variability between load-growth consequences under old and new units.

\section{Proposed Method for Evaluating the Transformer Price and Losses}

The power rating ( $M V A$ ) of DSPV transformers and replacement of units in service is undertaken instinctively on the basis of the technical or economical determination. Integrating these two aspects in the decision-making process can be even more rewarding for the IPP. In this work, new formulae for evaluating the transformer price and losses for IPPs are derived. These formulae will enable IPPs to optimally use existing units in service and establish a strategic plan to replace these units. Further, these formulae are critical when selecting a suitable power rating $(S)$ and the most economical service losses when purchasing new transformers. In the South African renewable energy market, there are a number of circumstances concerning the acquisition and replacement of transformers, including:

- Acquisition of a new transformer for the construction of a new solar project,

- Deferred replacement of transformer until end of designed service lifetime, and

- Superseding a unit in service with a larger rating.

In any case, the service lifetime of a transformer is reliant on its rating, loading profile and related annual growth. The option for IPPs to defer the replacement of transformers can only slow down the need for new capital investment, however, with the sacrifice of the service lifetime and higher cost of the service losses of the unit.

The derived formulae in this work are based upon empirical studies conducted on a fleet of transformers intended to be of service to various DSPV systems in South Africa that are ranging from 1.25MVA to 250MVA. The evaluation of the relationship between the transformer power rating against transformer prices, no-load losses and load losses is carried out by applying polynomial regression model.

\subsection{Polynomial Regression model}

Polynomial regression modelling has been chosen in this work to establish the relationship between various transformer ratings against the transformer price and the guaranteed design no-load and load losses.

$$
\hat{Y}=b_{0}+b_{1} x_{1}+b_{2} x_{2}{ }^{2}+\cdots+b_{k} x_{k}{ }^{k}
$$

here,

$\hat{Y}$ - Transformer anticipated outcome

$b_{0}-\mathrm{Y}$ intercept

$b_{1,2, \ldots k}-$ Regression model coefficients

$x_{1,2, \ldots k}-$ Regression model independent variables/predictor

A typical graphical representation of the polynomial regression model is shown in Figure 1.

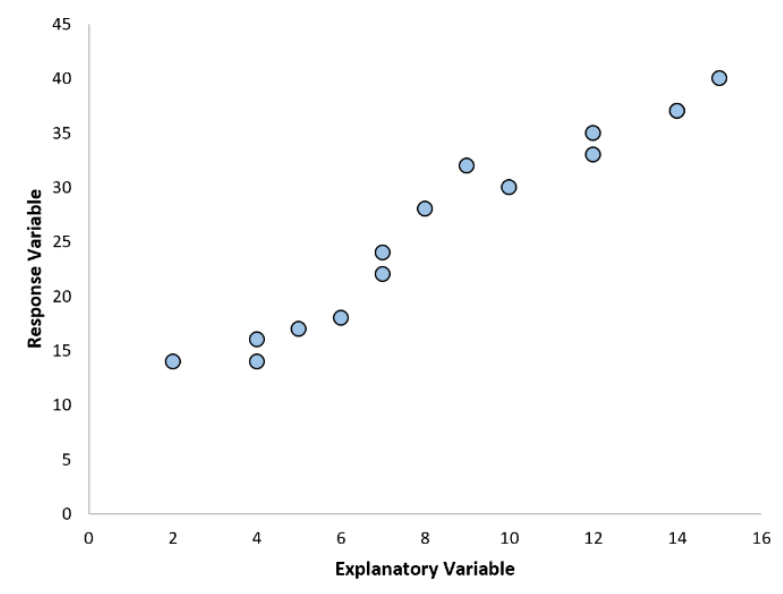

Figure 1: Typical Polynomial regression model

Some general proposition;

- The fitted data model is more credible when built on a fleet of transformer data.

- The transformer data is not extrapolated beyond the boundaries of observed data.

- The independent variables data selected are not too large as they will trigger overflow with higher order polynomials.

\subsection{Transformer Price (TP)}

The transformer price is dependent on various factors including ratings, materials and technical specification supplied by the customer, materials and manufacturing labour. A polynomial 
regression model using a quintic function to evaluate the cost of DSPV transformers is proposed and presented as follows in Eq. (6) below. This model is built upon a fleet of transformer price data that has been analysed using the general proposition presented in the previous subsection.

$T P=822069+371895 S-10435 \times S^{2}+139.4 S^{3}-0.758 S^{4}+1,39 e-3 \times S^{5}$

here,

\section{$T P$ - Transformer price in ZAR \\ $S$ - Transformer rating in MVA}

The rating in the studied transformers considers the highest voltage systems, top oil of $55^{\circ} \mathrm{C}$, mean winding temperature of $60^{\circ} \mathrm{C}$ and hotspot temperature of $78^{\circ} \mathrm{C}$. The relationship between the ratings the TP are demonstrated graphically as shown in Figure 2 .

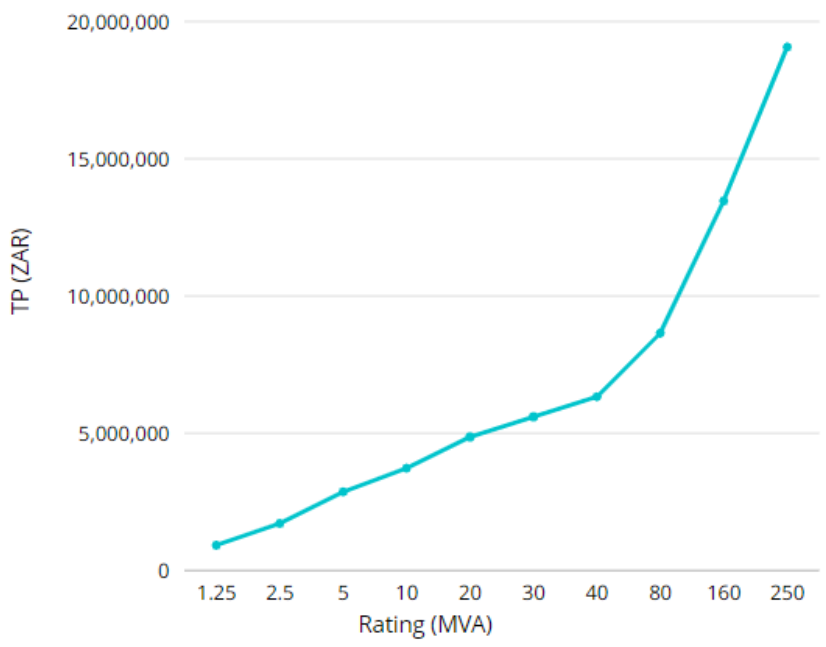

Figure 2: $M V A$ vs $Z A R$

The percentage contribution of the various components and materials that makes up the transformer price are summarised in Table 1 below. At the present time, the manufacturing of transformers is distinguished by a large range of designs required to meet a variety of customer with distinct technical requirements. Raw material prices will have an effect on these prices, but nonetheless, the percentage contribution will be somewhat as tabulated below.

Table 1: Total transformer manufacturing cost

\begin{tabular}{|l|c|}
\hline \multicolumn{1}{|c|}{ Item } & Percentage \\
\hline Bushings & $3 \%$ \\
\hline Tapchanger & $8 \%$ \\
\hline Cooling System & $7 \%$ \\
\hline Tank Steel & $4 \%$ \\
\hline Windings copper conductor & $17 \%$ \\
\hline Cellulose insulation & $5 \%$ \\
\hline Liquid insulation & $4 \%$ \\
\hline Transformer core & $5 \%$ \\
\hline Fittings and accessories & $5 \%$ \\
\hline Factory acceptance tests & $5 \%$ \\
\hline Manufacturing cost & $38 \%$ \\
\hline
\end{tabular}

The increase in the transformer price is observed to be reliant on the rating of the transformer. Another phenomenon is observed with regards to this relationship. In Eq. (7), the $Z A R / M V A$ function is also derived and presented as a quintic function.

$T P_{\frac{Z A R}{M V A}}=799349-53395 S+1605 S^{2}-21.58 S^{3}+0.101 S^{4}-2 e-4 S^{5}(7)$

Correspondingly, the graphical representation of this relationship in presented in Figure 3 below.

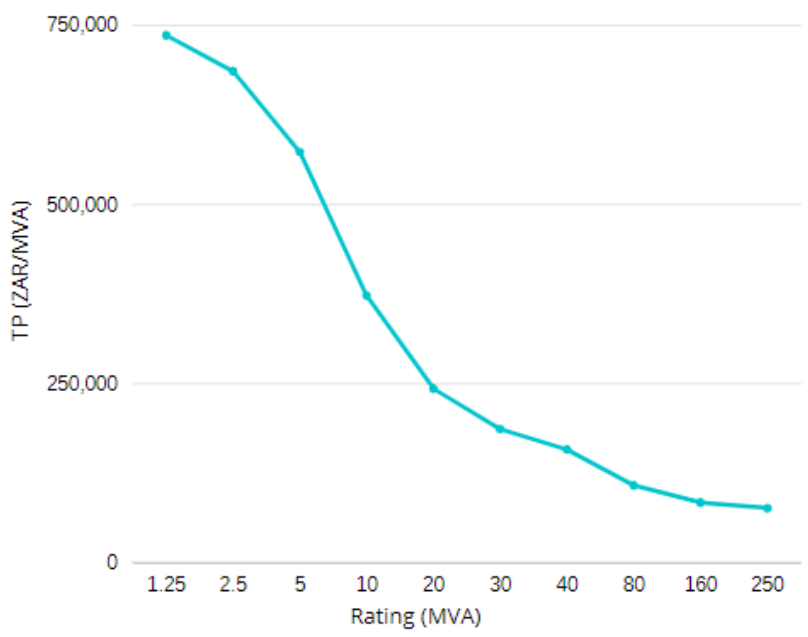

Figure 3: $M V A$ vs $Z A R / M V A$

It is observed the function $Z A R / M V A$ decreases as the rating of the transformer increase and slows down at about 160MVA and above.

\subsection{Transformer Losses}

The service no-load and load losses vary with the rating of the transformer. In the technical evaluation of competing bid offers, the service losses are critical parameters for the IPP. The quintic regression models derived for the no-load and load losses are expressed in Eq. (8) and Eq. (9) below.

$P_{N L}=2714+337.3 S+3.43 S^{2}-0.036 S^{3}+6,09 e-6 S^{4}+2.91 e-7 S^{5}$

$P_{L L}=-2302+7837 S-112 S^{2}+1.121 S^{3}-0.00567 S^{4}+1,04 e-5 S^{5}$

The relationship between the service losses and the rating is demonstrated graphically as shown in Figure 4.

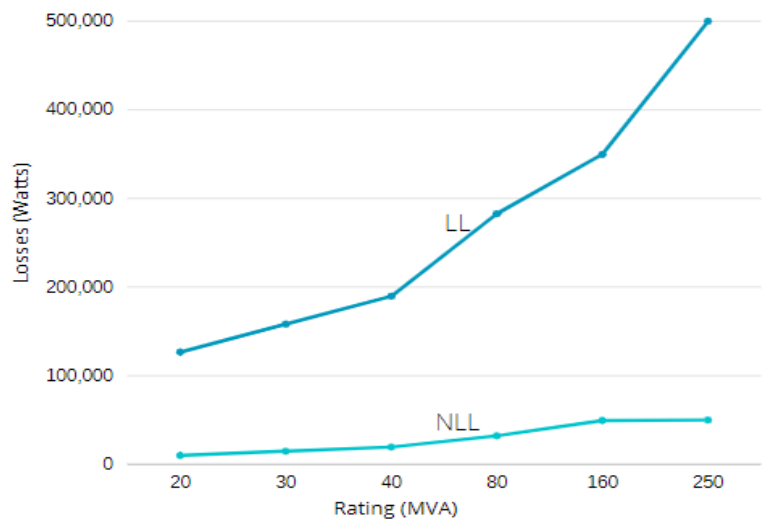

Figure 4: MVA vs Watts 
Correspondingly, the graphical representation of the relationship between the Watts/MVA and the rating is as demonstrated in Figure 5 below.

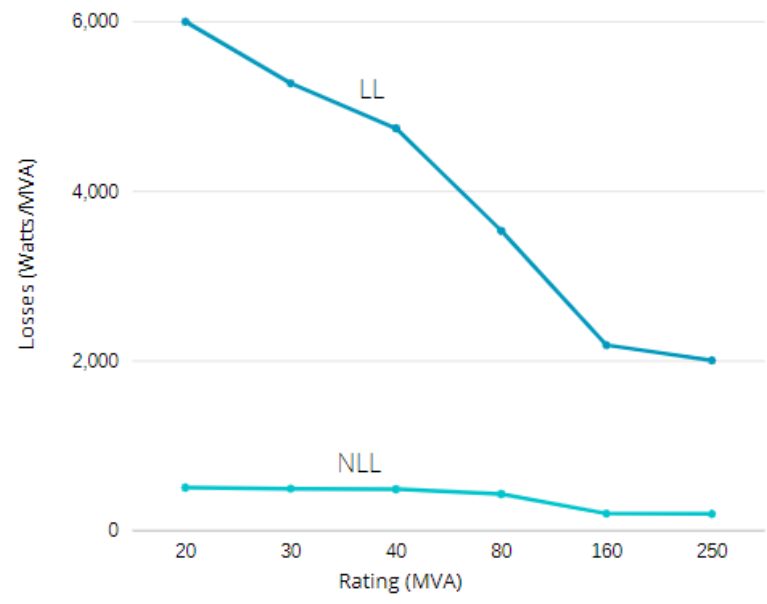

Figure 5: $M V A$ vs Watts/MVA

Finally, a set of new transformers intended for a DSPV project are studied in the next section to substantiate the new formulae.

\section{Case Scenarios}

In order to demonstrate the validity of the proposed method in this work, realistic techno-economic offers of various transformers have been provided subsequent to a request to bid issued by various IPPs within South Africa is presented in Table 2 below. The financial particulars considered in this case scenarios are adopted form our previous work [13].

Table 2: Bid offers

\begin{tabular}{|c|c|c|c|}
\hline$S$ & $T P$ & $P_{N L}$ & $P_{L L}$ \\
\hline 10 & 3729213 & 6437 & 65506 \\
\hline 20 & 4864152 & 10541 & 117661 \\
\hline 40 & 6332296 & 19533 & 189746 \\
\hline 160 & 13468698 & 32230 & 349712 \\
\hline
\end{tabular}

In the next sub-section, the assessment of these offers is carried out for the individual sizes.

\subsection{Application and Validation of Proposed Method}

To facilitate a bona fide comparison of the proposed (prop) noload and load loss formulae against supplied data in Table 2, Eq. (8) and Eq. (9) are applied to estimate these losses as indicated in Table 3.

Table 3: Benchmarking of no-load and load losses

\begin{tabular}{|l|c|c|c|c|c|c|}
\hline$S$ & $P_{N L(A c t)}$ & $P_{N L(\text { Prop })}$ & Error & $P_{L L(A c t)}$ & $P_{L L(\text { Prop })}$ & Error \\
\hline 10 & 6437 & 6394 & 0,67 & 65506 & 65916 & 0,63 \\
\hline 20 & 10541 & 10546 & 0,05 & 117661 & 117667 & 0,00 \\
\hline 40 & 19533 & 19441 & 0,47 & 189746 & 190020 & 0,14 \\
\hline 160 & 32230 & 32229 & 0,00 & 349712 & 349714 & 0,00 \\
\hline
\end{tabular}

The results indicate that the new formulae have an error of estimation of less than $1 \%$ in all cases. The proposed loss evaluation procedure by the authors and associated annualised cost of energy, interest rate, service lifetime and the generating and non-generating state factors have been adopted from a previous work [13] in order to evaluate the cost of service losses and TOC of the studied transformers. The TOC of the units based upon the actual values is evaluated and tabulated as shown in Table 4 below. In our previous work [13], we have derived harmonic loss factors of DSPV transformers in which IPPs can apply to evaluate the cost of $C_{N L}$ and $C_{L L}$ as shown below. Additionally, the TOC is evaluated using Eq. (5) as expressed in section sub-section 2.1 above. Hence, Table 4 tabulates the calculated TOC of individual transformer sizes described in Table 2.

Table 4: Assessment of bid offers (Actual)

\begin{tabular}{|l|c|c|c|}
\hline$S$ & $C_{N L}$ & $C_{L L}$ & TOC \\
\hline 10 & 222697 & 1389795 & 5341705 \\
\hline 20 & 364680 & 2496331 & 7725164 \\
\hline 40 & 675771 & 4024987 & 11033054 \\
\hline 160 & 1115040 & 7419596 & 22003334 \\
\hline
\end{tabular}

On the other hand, the TOC of the units based upon the new formulae is evaluated and tabulated as shown in Table 5 below.

Table 5: Assessment of bid offers (Proposed)

\begin{tabular}{|l|c|c|c|}
\hline$S$ & $C_{N L}$ & $C_{L L}$ & TOC \\
\hline 10 & 221209 & 1389795 & 5340217 \\
\hline 20 & 364853 & 2496331 & 7725337 \\
\hline 40 & 499606 & 4025709 & 10857610 \\
\hline 160 & 1115006 & 7419638 & 22003342 \\
\hline
\end{tabular}

The error of estimation for Table 4 and Table 5 above is tabulated as shown in Table 6 below.

Table 6: Assessment of bid offers (Comparison)

\begin{tabular}{|l|c|c|c|}
\hline$S$ & $C_{N L}$ & $C_{L L}$ & $T O C$ \\
\hline 10 & $0,67 \%$ & $0,00 \%$ & $0,03 \%$ \\
\hline 20 & $0,05 \%$ & $0,00 \%$ & $0,00 \%$ \\
\hline 40 & $1,37 \%$ & $0,02 \%$ & $1,59 \%$ \\
\hline 160 & $0,00 \%$ & $0,00 \%$ & $0,00 \%$ \\
\hline
\end{tabular}

The results yield an error of estimation of less than $2 \%$ in all of the studied transformer ranges. It is recommendable that IPPs use the new formulae in this work when planning to deploy new solar projects to indicate the cost of the units, losses, cost of operation, and the TOC.

\section{Conclusion}

It is well documented that the prevailing factors in evaluating the service lifetime cost of DSPV transformers are the power rating and the associated service losses. These components need to be adjusted when major considerations such as rates of exchange for materials - provided that they affect the overall acquisition of materials and manufacturing cost.

More particularly, this work defines new formulae to determine the techno-economic parameters of DPSV transformers within the South African renewable energy market by deriving the contribution of the transformer price and the service losses to the TOC. In previous work, a method to evaluate the TOC was 
proposed. This method and associated price indexes are applied in the current work to determine the cost of no-load, load loss and Total Ownership Cost of various transformers. The results yield an error of estimation of $2 \%$ against actual values for all the studied costs.

Future work will explore the development of regression models for evaluating the cost of losses and the TOC.

\section{Conflict of Interest}

The authors declare no conflict of interest.

\section{References}

[1] 6Wresearch, "South Africa Power \& Distribution Transformer Market (2020-2026), No. 36005, May 2020".

[2] S. Mamphweli, "South Africa's renewable energy landscape", February 2019.

[3] NDC Partnership," Making renewable energy affordable: The South African Renewables Initiative", ndcpartnership.org/case-study/making-renewableenergy-affordable-south-african-renewables-initiative

[4] B. A. Thango, J. A. Jordaan and A. F. Nnachi, "Effects of Current Harmonics on Maximum Loading Capability for Solar Power Plant Transformers," 2020 International SAUPEC/RobMech/PRASA Conference, Cape Town, South Africa,2020,doi:10.1109/SAUPEC/RobMech/PRASA48453.2020.9041101

[5] B. A. Thango, J. A. Jordaan and A. F. Nnachi, "Step-Up Transformers for PV Plants: Load Loss Estimation under Harmonic Conditions," $202019^{\text {th }}$ International Conference on Harmonics and Quality of Power (ICHQP), Dubai, United Arab Emirates, 2020, doi: 10.1109/ICHQP46026.2020.9177938.

[6] D. Pavlik, D. C. Johnson and R. S. Girgis, "Calculation and reduction of stray and eddy losses in core-form transformers using a highly accurate finite element modelling technique," in IEEE Transactions on Power Delivery, 8(1), Jan. 1993, doi: 10.1109/61.180342.

[7] X. Yan, X. Yu, M. Shen, D. Xie and B. Bai, "Research on Calculating EddyCurrent Losses in Power Transformer Tank Walls Using Finite-Element Method Combined with Analytical Method," in IEEE Transactions on Magnetics, 52(3), March 2016, doi: 10.1109/TMAG.2015.2494375.

[8] B. A. Thango, J. A. Jordaan and A. F. Nnachi, "Service Life Estimation of Photovoltaic Plant Transformers Under Non-Linear Loads," 2020 IEEE PES/IAS PowerAfrica, Nairobi, Kenya, 2020, doi: 10.1109/PowerAfrica49420.2020.9219912.

[9] E. Müllerová, J. Hrůza, J. Velek, I. Ullman and F. Stř́ska, "Life cycle management of power transformers: results and discussion of case studies," in IEEE Transactions on Dielectrics and Electrical Insulation, 22(4), August 2015, doi: 10.1109/TDEI.2015.005025.

[10] Q. Guo-Hua, R. Zheng, S. Lei, Z. Bo, X. Jian-Gang and Z. Xiang-Ling, "A new life cycle cost model of power transformer and its comprehensive sensitivity analysis," 2014 International Conference on Power System Technology, Chengdu, 2014, doi: 10.1109/POWERCON.2014.6993659.

[11] D. Ambach and P. Vetter, "Wind Speed and Power Forecasting - A Review and Incorporating Asymmetric Loss," 2016 Second International Symposium on Stochastic Models in Reliability Engineering, Life Science and Operations Management (SMRLO), Beer-Sheva, 2016, doi: 10.1109/SMRLO.2016.29.

[12] R. S. Tarade and P. K. Katti, "A comparative analysis for wind speed prediction," 2011 International Conference on Energy, Automation and Signal, Bhubaneswar, Odisha, 2011, doi: 10.1109/ICEAS.2011.6147167.

[13] B. A. Thango, J. A. Jordaan and A. F. Nnachi, "Total Ownership Cost Evaluation for Transformers within Solar Power Plants," $20206^{\text {th }}$ IEEE International Energy Conference (ENERGYCon), Gammarth, Tunis, Tunisia, 2020, doi: 10.1109/ENERGYCon48941.2020.9236613.
[14] J. F. Baranowski and P. J. Hopkinson, "An alternative evaluation of distribution transformers to achieve the lowest TOC," in IEEE Transactions on Power Delivery,7(2), April 1992, doi: 10.1109/61.127057.

[15] IEEE Std. C57.120-2017, "IEEE Guide for Loss Evaluation of Distribution and Power Transformers and Reactors," in IEEE Std C57.120-2017 (Revision of IEEE Std C57.120-1991), 18 Oct. 2017, doi: 10.1109/IEEESTD.2017.8103991.

[16] H. Wen, H. Zou, C. Ouyang, X. Bao, H. Li and X. Li, "Exploration on energy-saving effect of amorphous transformers extended in Nanning based on theoretical analysis and Total Owning Cost methods (TOC)," 2008 China International Conference on Electricity Distribution, Guangzhou, 2008, doi: 10.1109/CICED.2008.5211678.

[17] B. A. Thango, J. A. Jordaan and A. F. Nnachi, "Contemplation of Harmonic Currents Loading on Large-Scale Photovoltaic Transformers," $20206^{\text {th }}$ IEEE International Energy Conference (ENERGYCon), Gammarth, Tunis, Tunisia, 2020, doi: 10.1109/ENERGYCon48941.2020.9236514.

[18] B. A. Thango, J. A. Jordaan and A. F. nnachi, "A Weighting Factor for Estimating the Winding Eddy Loss in Transformers for High Frequencies," $20206^{\text {th }}$ IEEE International Energy Conference (ENERGYCon), Gammarth, Tunis, Tunisia, 2020, doi: 10.1109/ENERGYCon48941.2020.9236472. 